\title{
Extension Neural Network Optimized by Election Campaign Algorithm for Fault Diagnosis
}

\author{
Qinghua Xie ${ }^{1, a^{*}}$, Xiangwei Zhang ${ }^{1, b}$, Wenge $\mathrm{Lv}^{1, \mathrm{c}}$ and Siyuan Cheng ${ }^{1, \mathrm{~d}}$ \\ ${ }^{1}$ School of Electromechanical Engineering, Guangdong University of Technology, Guangzhou china \\ axqhcool@163.com, bxwzhang@gdut.edu.cn, 'Ivwenge@163.com, dimdesign@gdut.edu.cn
}

Keywords: Fault diagnosis; Extension neural network; matter element; Optimization; Election campaign algorithm

\begin{abstract}
Extension fault diagnosis is a new research direction in the field of intelligent fault diagnosis. The extension neural network model is introduced, including its structure and diagnostic principle. But for the problems of subjective parameters setting and algorithm precocious, the extension neural network model based on election campaign algorithm is proposed. It takes the dependent degree as the measurement and optimizes the parameters by using election campaign algorithm. The results of experiment show that using this algorithm the entire fault can be correctly detected and the precision is high.
\end{abstract}

\section{Introduction}

Artificial neural network is a kind of artificial neural network behavior characteristic, and it is a mathematical model of distributed parallel information processing. This network relies on the complexity of the system, through the adjustment of the internal relationship between nodes, so as to achieve the purpose of processing information. Artificial neural network has the ability of self-learning and adaptive, can be provided in advance a number of mutually corresponding input / output data analysis to grasp the potential between the two laws, finally according to these rules, with new input data to calculate the output results. Artificial neural network has the unique nonlinear adaptive information processing ability, to overcome the traditional methods of artificial intelligence for intuition, such as pattern, speech recognition and non-structured information processing, which has been successfully applied in neural expert system, pattern recognition, intelligent control, classification and clustering, combinatorial optimization, prediction and other fields.

Combining artificial neural network with other methods will promote the development of artificial intelligence and information processing technology. For example, in recent years, the combination of artificial neural network and fuzzy mathematics, genetic algorithm and evolution mechanism, the formation of intelligent computing, and artificial neural network forward simulation of human cognition more in-depth development, has become an important direction of artificial intelligence.

Extenics is a cross discipline founded by Professor Cai in 1983 [1]. It proposes math language to research things of development [2]. With extension innovation rules and methods, it resolves mainly the conflict problems [3]. Extenics has been applied to the economic, management, decision-making, information technology, new product design and intelligent control. Extension neural network is a new type of network after the fuzzy neural network, genetic neural network, and evolutionary neural network $[4,5]$.

With the extension theory and the improvement of neural network, extension neural network is gradually become a new hotspot in classification and clustering, pattern recognition, fault diagnosis, detection and other aspects application, and achieved good results [6-9].

The basic method of extension fault diagnosis is superior degree evaluation method. The advantage of this method is simple and direct and easy to understand. The disadvantage is that the classical domain, the nodal region and the weight coefficient are determined subjectively. Based on this, some scholars proposed extension neural network with dual weight link structure. This method uses the upper and lower bounds of the classical domain as the double weight of the neural network, and ignores the function of the joint region. Using the extension distance as the measure tool, the 
algorithm cannot get the optimal solution. An extension neural network based on election campaign algorithm optimization is proposed in this paper. It optimizes the parameters by using election campaign algorithm, so that the extension distance could work and the extension neural network be build.

\section{Correlation Function}

In this paper, we use the elementary correlation function as the measure tool of the input node and the neuron in the extension neural network.

If set $x$ to any point on the real axis, $X_{0}=\langle a, b\rangle$ is any interval on the real field

$$
\rho\left(x, X_{0}\right)=\left|x-\frac{a+b}{2}\right|-\frac{b-a}{2}
$$

The distance between point $x$ and interval $X_{0}$ is calculated by Eq. 1 .

If set $X=\langle c, d\rangle, X_{0}=\langle a, b\rangle$ and $X_{0} \subset X$ is intervals on the real field, then any point $x$ on the real axis on the location of the interval sets of $X_{0}$ and $X$ is specified as Eq. 2. $D\left(x, X_{0}, X\right)$ describes the location of the point $x$ and the components of the interval sets $X_{0}$ and $X$.

$$
\begin{aligned}
& \text { when } \quad \rho(x, X) \neq \rho\left(x, X_{0}\right) \\
& D(x, X 0, X)=\left\{\begin{array}{r}
\rho(x, X)-\rho\left(x, X_{0}\right), x \in X_{0} \\
\rho(x, X)-\rho\left(x, X_{0}\right)+a-b, x \in X_{0}
\end{array}\right. \\
& \text { when } \rho(x, X)=\rho\left(x, X_{0}\right) \\
& D(x, X 0, X)=a-b
\end{aligned}
$$

If set $\mathrm{X}=\left\langle\mathrm{c}, \mathrm{d}>, \mathrm{X} 0=<\mathrm{a}, \mathrm{b}>\right.$ and $\mathrm{X} 0 \subset \mathrm{X}$ is intervals on the real field, common endpoint $x_{z}$ of the interval sets of $X_{0}$ and $X$ is specified as Eq. 3. If there is no common endpoint, $x_{z}$ is null. For any point $x \neq x_{z}$ in $X_{0}, k(x)$ is the elementary correlation function at the point $x$ of the maximum value of $X_{0}$ and $X$.

$$
\begin{aligned}
& \text { when } \rho(x, X)=\rho\left(x, X_{0}\right) \text { and } x \notin X_{0} \\
& k(x)=\frac{\rho\left(x, X_{0}\right)}{D\left(x, X_{0}, X\right)}-1 \\
& \text { else }
\end{aligned}
$$

$$
k(x)=\frac{\rho\left(x, X_{0}\right)}{D\left(x, X_{0}, X\right)}
$$

\section{Extension Neural Network}

In this paper, the extension neural network is a kind of feedforward network, which has only one layer of neurons. Fault feature vector input node corresponding to the extension of the neural network, the output nodes corresponding to each fault type, neurons and the output nodes corresponding to each neuron contains a summation function and a transfer function.

Fig. 1 is the extension neural network structure of general single link weight connection. The circle in the figure are neurons, usually it is a matter element $R=(N, C, V)$. The lines between matter element neurons indicates the connection between neurons. Connection weights $w$ determined by the correlation function. ENN consists of a large number of extension neurons which are connected with each other to form a single layer or multi-layer network topology. It obtains the information from the outside through the material element input layer, after the internal operation of the network (the matter element transformation, the extension set, the operation of the correlation function, etc.), the output set is delivered to the network.

Extension neural network is a new type of neural network. The main idea of extension neural network is: extension neural network is the extension element theory model, extension distance and place value, correlation function, extension field, rhombus thinking concepts such as introducing 
neural network technology, which has more advantages in the treatment of a class compared with the traditional neural network problems or single use of extension theory method

According to the actual use of stone cutting precision, we use virtual grid graphics witch has the spacing of required precision in the image. Each pixel of image is divide to equal virtual grid just like the sub pixel method ${ }^{[6]}$. A sample profile retrieval each virtual grid in the region, the definition of each gird is 0 , gird out of the region is not 0 . For example, the actual graphic discretization is shown in Fig. 1. For the interpretation of clarity, we abstract some exceptions for sample enlarged graphics. If you can retrieve the picture element in the virtual grid area and small area, is defined as 0 , said the need to discharge at the corresponding position; the remaining non 0, as shown in Figure 1 (b). Fig. 1 (c) said there is a hole in the middle of the picture. All discharge can be considered as the target number is non-zero.

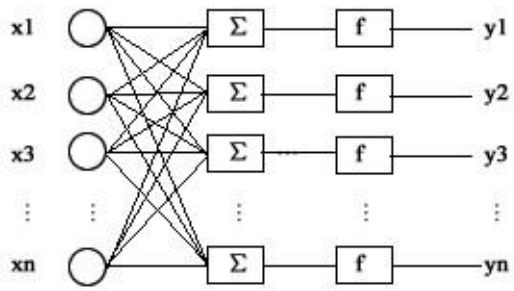

Figure 1. Structure of Extension Neural Network

If set a training sample $O=\left\{O_{t}, t=1,2, \cdots, T\right\}$, each sample $O_{t}$ has $n$ Characteristic index $c_{1}, c_{2}, \cdots$; $c_{n}$, the corresponding value of each characteristic index is $x_{1}, x_{2}, \cdots, x_{n}$, the matter element model for each sample $O_{t}$ is Eq. 4.

$$
M_{t}=\left(O_{t}, C, X\right)=\left[\begin{array}{ccc}
O_{t} & c_{1} & x_{1}(t) \\
& c_{2} & x_{2}(t) \\
& \vdots & \vdots \\
& c_{n} & x_{n}(t)
\end{array}\right]
$$

Set the training sample $O$ possible fault set is $F=\left\{F_{1}, F_{2}, \cdots, F_{m}\right\}$, the classical domain matter element model for each fault $F_{i}$ is Eq. 5 . When the fault occurs in the sample $F_{i}$, the classical domain of the characteristic index $c_{j}$, the lower and upper bound are $x^{C L}$ and $x^{C U}$.

$$
\begin{aligned}
& M_{i}^{C}=\left(F_{i}, C, X_{i}^{C}\right)=\left[\begin{array}{ccc}
F_{i} & c_{1} & X_{i 1}^{C} \\
& c_{2} & X_{i 2}^{C} \\
& \vdots & \vdots \\
& c_{n} & X_{i n}^{C}
\end{array}\right] \\
& X_{i j}^{C}=<x_{i j}^{C L}, x_{i j}^{C U}>
\end{aligned}
$$

For the whole fault set $\mathrm{F}$, the matter element model of the whole fault set is Eq.6. Fault set $F$ the characteristic index $c_{j}$ of the node domain

$$
\begin{aligned}
& M^{S}=\left(F_{i}, C, X^{S}\right)=\left[\begin{array}{ccc}
F_{i} & c_{1} & X_{1}^{S} \\
& c_{2} & X_{2}^{S} \\
& \vdots & \vdots \\
& c_{n} & X_{n}^{S}
\end{array}\right] \\
& X_{j}^{S}=<x_{j}^{S L}, x_{j}^{S U}>=<\min _{t=1,2, \cdots, T}\left\{x_{j}(t)\right\}, \max _{t=1,2, \cdots, T}\left\{x_{j}(t)\right\}>, j=1,2, \cdots, n
\end{aligned}
$$

The value of any matter element $c_{j}$ is $x_{j}(t)$, correlation degree of the classical field matter element and the same characteristic field element in each fault $F_{i}$ is showed in Eq. 7. 


$$
\begin{aligned}
& \text { when } \quad \rho\left(x_{j}(t), X_{i j}^{C}\right)=\rho\left(x_{j}(t), X_{j}^{S}\right) \text { and } x_{j}(t) \notin X_{i j}^{C} \\
& k_{i j}(t)=\frac{\rho\left(x_{j}(t), X_{i j}^{C}\right)}{D\left(x_{j}(t), X_{i j}^{C}, X_{j}^{S}\right)}-1
\end{aligned}
$$

else

$$
k_{i j}(t)=\frac{\rho\left(x_{j}(t), X_{i j}^{C}\right)}{D\left(x_{j}(t), X_{i j}^{C}, X_{j}^{S}\right)}
$$

The neuron $N_{i}$ is corresponding to the fault $F_{i}$, and the correlation degree $K_{i}(t)$ of $F_{i}$, is the input of neuron $N_{i}$. The weight coefficient $\alpha_{i j}$ of the characteristic index $c_{j}$ to the fault $F_{i}$.

$$
K_{i}(t)=\sum_{j=1,2, \cdots, n} \alpha_{i j} k_{i j}(t), \quad \sum_{j=1,2, \cdots, n} \alpha_{i j}=1
$$

According to the correlation function, correlation requirements $K_{i}(t)$ of sample $M_{t}$ corresponding to the fault $F_{i}$ must be $K_{i}(t) \geq 0$. The fault type $T_{f}$ of sample element $M_{t}$ is showed in Eq. 10 . When $\left|T_{f}\right|=1$, matter element is single fault. When $\left|T_{f}\right|>1$, matter element is multiple faults. When $\left|T_{f}\right|=0$, matter element is not in the $F$.

$$
\begin{aligned}
& y_{i}(t)=f\left(K_{i}(t)\right)=\left\{\begin{array}{l}
1, K_{i}(t) \geq 0 \\
0, K_{i}(t)<0
\end{array}\right. \\
& T_{f}=\left\{F_{j} \mid y_{j}(t)==1\right\}
\end{aligned}
$$

\section{Election Campaign Algorithm for Link Weight Optimization}

Election campaign algorithm (ECA) is an optimization algorithm simulating election process [10]. Framework of the election campaign algorithm is described as the follows:

\section{Set the Algorithm Parameters.}

Generate the Candidates and Calculate the Prestige of Them. Object function is the correlation degree $K_{i}(t)$ of $F_{i}$.

\section{Generate the Voters.}

Compute the Investigate Mean Square of Candidates.

Calculate the Supported of the Voters. The support from the voter $w_{j}$ to $w_{i j}$ is calculated by Eq. 11.

$$
w_{i j}=\frac{E_{i j}}{\sum_{i=1}^{m} \max \left(E_{1 j}, E_{2 j}, \cdots, E i j, \cdots E_{m j}\right)} w_{j}
$$

Find the Support Focus of Candidates. $w$ is the inertia weight and the focus center. It is calculated by Eq. 12 .

$$
w=\frac{\sum_{j=1}^{n} S_{i j} x_{j}}{\sum_{j=1}^{n} \max \left(S_{i 1}, S_{i 2}, \cdots, S_{i j}, \cdots S_{i n}\right)}
$$

Calculate the Prestige of the Candidates and Compare the Voters with the Candidates. Check Whether the Condition is Reach. Check whether the iterative is complete.

\section{Experiment}

Reference to the sample data in [11], 58 sets of data are selected to set up the fault set of transformer fault diagnosis. The sample distribution of the fault set is shown in Tab. 1.

Using Election campaign algorithm, the number of candidates define as 5, the number of voters is 10 , the number of floating voters is 5 , and the target accuracy is set as $1 \%$. 
Table 1 Distribution of fault samples

\begin{tabular}{|c|c|c|c|c|c|}
\hline [fault type] & $\begin{array}{c}\text { low temperature } \\
\text { overheat }\end{array}$ & $\begin{array}{c}\text { middle } \\
\text { temperature } \\
\text { overheat }\end{array}$ & $\begin{array}{c}\text { high } \\
\text { temperature } \\
\text { overheat }\end{array}$ & $\begin{array}{c}\text { low energy } \\
\text { discharge }\end{array}$ & $\begin{array}{c}\text { high energy } \\
\text { discharge }\end{array}$ \\
\hline [sample number] & 7 & 14 & 12 & 13 & 10 \\
\hline
\end{tabular}

The experiment achieve the correct division of the transformer fault diagnosis, it prove the feasibility of the algorithm.

\section{Conclusions}

This paper uses correlation as a measure tool of extension neural network, and the extension neural network weights optimization using particle election campaign algorithm. The experiments show that this method can correctly diagnose the faults of the tested sample and has not the disadvantage of other fault diagnosis method.

\section{References}

[1] Wen Cai, Extension theory and its applications, Chinese Science Bulletin, 1999, 44(17), pp. 1538-1548.

[2] Yongquan Yu, Haixia Peng, The principle of extension detecting with extension sets, Proc of International Conference on Computer, Communication and Control Technologies, Orlando, INTINST Informatics \& Systemics, 2003, pp. 113-118.

[3] M. H. Wang, Y. F. TSENG, A novel clustering algorithm based on the extension theory and genetic algorithm, Expert Systems with Applications, 2009,36(4), pp. 8269-8276.

[4] M. H. Wang, Application of extension theory to PD pattern recognition in high-voltage current transformers, IEEE Trans on Power Delivery, 2005, 20(3), pp. 1939-1946.

[5] Jun Ye, Application of extension theory in misfire fault diagnosis of gasoline engines, Expert Systems with Applications, 2009, 36(2), pp. 1217-1221.

[6] S. Mohamed, T. Tettey, T. Marwala, An extension neural network and genetic algorithm for bearing fault classification, Proc of International Joint Conference on Neural Networks. 2006, pp. 3941-3948.

[7] B. Vilakazic, T. Marwala, Bushing fault detection and diagnosis using extension neural network, Proc of International Conference on Intelligent Engineering Systems. 2006, pp. 170-174.

[8] B. Vilakazic, T. Marwala, Agents and multi-agent systems and application to condition monitoring, Proc of IEEE International Conference on Systems, Man and Cybernetics. 2007, pp. 644-649.

[9] Y. H. Lai, C. Chen. Modeling patent legal value by extension neural network, Expert Systems with Applications, 2009, 36(7), pp. 10520-10528.

[10] Wenge Lv, Chunhua He, DeyuanLi, et al., Election Campaign Optimization Algorithm. Procedia Computer Science, 1, pp. 1371-1380.

[11]Jian Li, Caixin Sun, Weigen Chen, A method of synthesis based on the grey cluster and fuzzy cluster about internal fault diagnosis of transformer, Proceedings of the CSEE. 2003, 3(2) pp. 112-I15. 